\title{
Segurança e Benefício do Uso de Probióticos em Pacientes Submetidos ao TCTH:Revisão Integrativa
}

doi: https://doi.org/10.32635/2176-9745.RBC.2019v65n4.14

\author{
Safety and Benefit of Using Probiotics in Patients Undergoing HSCT: Integrative Review \\ Seguridad y Beneficio del Uso de Probióticos en Pacientes Sometidos al TCTH: Revisión Integradora
}

Paola Dantas Pinheiro de Oliveira'; Denise Johnsson Campos²; Vaneuza Araújo Moreira Funke³ ${ }^{3}$ Sarah Mehl Coradi Regina Maria Vilela ${ }^{5}$

Resumo

Introdução: $\mathrm{O}$ transplante de células-tronco hematopoiéticas (TCTH) é um dos potenciais tratamentos curativos utilizados para pacientes com doenças hematológicas e outras doenças imunes. Durante o transplante, o paciente é submetido ao condicionamento e a outros tratamentos, como radioterapia e quimioterapia, o que pode causar a perda da diversidade da microbiota intestinal. A manipulação da microbiota intestinal com probióticos vem sendo apontada como uma estratégia de prevençâo de complicaçôes nos pacientes submetidos ao TCTH. Objetivo: Identificar se há evidências científicas relacionadas à segurança e aos benefícios da utilização de probióticos em pacientes submetidos ao TCTH. Método: Revisão integrativa com base em estudos que abordassem o uso de probióticos para o caso específico de pacientes submetidos ao TCTH publicados entre 2000 a 2018. Resultados: Foram selecionados cinco estudos que atenderam aos critérios de inclusão e exclusão, com um total de 52 pacientes. A utilização de probióticos na prevenção e/ou tratamento da diarreia tem mostrado resultados positivos em pacientes com diarreia induzida por antibióticos ou por infecçóes bacterianas, porém os estudos ainda não destacam benefícios no uso de probióticos no caso específico de pacientes submetidos ao TCTH. Poucos estudos mostram o uso de probióticos para auxílio na melhora dos sintomas associados a infecçôes ou bacteremias em pacientes imunossuprimidos. Conclusão: $\mathrm{O}$ uso de probióticos na população submetida ao TCTH e em imunossuprimidos ainda é controverso, sendo necessários mais estudos que demonstrem os benefícios no uso dessa estratégia para esse público.

Palavras-chave: Probióticos; Transplante de Células-Tronco Hematopoéticas; Hospedeiro Imunocomprometido; Microbioma Gastrointestinal; Imunidade.

\begin{abstract}
Introduction: Hematopoietic stem cell transplantation (HSCT) is one of the potential curative treatments used for patients with hematological and other immune diseases. During transplantation, the patient undergoes conditioning and other treatments, such as radiotherapy and chemotherapy, which may cause loss of the intestinal microbiota diversity. The manipulation of the intestinal microbiota with probiotics has been pointed out as a strategy to prevent complications in patients undergoing HSCT. Objective: To identify if there is scientific evidence related to the safety and benefits of the use of probiotics in patients submitted to HSCT. Method: Integrative review based on studies addressing the use of probiotics for the specific case of patients undergoing HSCT published between 2000 and 2018. Results: Five studies that met the inclusion and exclusion criteria were eligible, with a total of 52 patients. The use of probiotics in the prevention and/or treatment of diarrhea has shown positive results in patients with antibiotic-induced diarrhea or bacterial infections, but the studies do not yet emphasize the benefits of using probiotics in the specific case of patients submitted to HSCT. Few studies show the use of probiotics to help the improvement of the symptoms associated to infections or bacteremia in immunosuppressed patients. Conclusion: The use of probiotics in the population submitted to HSCT and immunosuppressed is still controversial, and further studies are necessary to demonstrate the benefits of using probiotics for this public. Key words: Probiotics; Hematopoietic Stem Cell Transplantation; Immunocompromised Host; Gastrointestinal Microbiome; Immunity.
\end{abstract}

Resumen

Introducción: El trasplante de células madre de las hematopoyéticas (TCTH) es uno de los posibles tratamientos curativos utilizados para pacientes con enfermedades hematológicas y otras enfermedades inmunes. Durante el transplante, el paciente es sometido al condicionamiento ya otros tratamientos, como radioterapia y quimioterapia, lo que puede causar la pérdida de la diversidad de la microbiota intestinal. La manipulación de la microbiota intestinal con probióticos viene siendo apuntada como una estrategia de prevención de complicaciones en los pacientes sometidos al TCTH. Objetivo: Identificar si hay evidencias científicas relacionadas con la seguridad y beneficios de la utilización de probióticos en pacientes sometidos al TCTH. Método: Revisión integradora basada em estúdios que abordan el uso de probióticos para el caso específico de pacientes sometidos a TCMH publicados entre 2000 y 2018. Resultados: Fueron elegibles 4 estudios que atendieron a los criterios de inclusión y exclusión, con un total de 52 pacientes. La utilización de probióticos en la prevención y/o tratamiento de la diarrea ha mostrado resultados positivos en pacientes con diarrea inducida por antibióticos o por infecciones bacterianas, pero los estudios aún no aportan beneficios en el uso de probióticos en pacientes sometidos al TCTH. Pocos estudios muestran infecciones o bacterias en pacientes inmunosuprimidos que utilizaron probióticos para ayudar en la mejora de los síntomas asociados al tratamiento. Conclusión: El uso de probióticos en la población sometida al TCTH e inmunosuprimidos aún es controvertido, siendo necesarios más estudios que comprueben los beneficios en el uso de probióticos para este público.

Palabras clave: Probióticos; Trasplante de Células Madre Hematopoyéticas; Huésped Inmunocomprometido; Microbioma Gastrointestinal; Inmunidad.

\footnotetext{
${ }^{1}$ Hospital de Clínicas da Universidade Federal do Paraná (UFPR). Curitiba (PR), Brasil. Orcid iD: https://orcid.org/0000-0003-2311-7298

${ }^{2}$ Hospital de Clínicas da UFPR. Curitiba (PR), Brasil. Orcid iD: https://orcid.org/0000-0001-9255-2352

${ }^{3}$ Hospital de Clínicas da UFPR. Curitiba (PR), Brasil. Orcid iD: https://orcid.org/0000-0002-2122-7277

${ }^{4}$ Hospital de Clínicas da UFPR. Curitiba (PR), Brasil. Orcid iD: https://orcid.org/0000-0002-1734-5692

${ }^{5}$ Hospital de Clínicas da UFPR. Curitiba (PR), Brasil. Orcid iD: https://orcid.org/0000-0003-0716-1643

Endereço para correspondência: Regina Maria Vilela. Avenida Prefeito Lothário Meissner, 632 - Jardim Botânico. Curitiba (PR), Brasil. CEP 80210-170.

E- mail: regina.vilela@mail.mcgill.ca
} 


\section{INTRODUÇÃO}

O transplante de células-tronco hematopoiéticas (TCTH) é um dos potenciais tratamentos curativos utilizados para pacientes com doenças hematológicas malignas e outras doenças ${ }^{1}$. No período pré-TCTH, o paciente é submetido ao regime de condicionamento e a outros tratamentos, como quimioterapia e radioterapia, que têm como finalidade erradicar a doença residual ${ }^{2}$, depletando tanto as células malignas ou deficientes quanto as células saudáveis ${ }^{1}$. Nesse período, ocorre a perda da diversidade da microbiota intestinal, o que vem sendo apontado como importante fator de aumento de morbidade e mortalidade pós-TCTH ${ }^{3}$. Estudos sugerem que, durante o período de neutropenia, as espécies presentes na microbiota intestinal decresçam aproximadamente $30 \%$ se comparado ao período pré$-\mathrm{TCTH}^{4}$.

Atualmente, estudos apontam que a manipulação da microbiota intestinal com probióticos poderia ser benéfica em pacientes submetidos ao TCTH, a fim de reduzir a morbidade causada pela perda da diversidade microbiana ${ }^{4}$. No entanto, a manipulação da microbiota intestinal com uso de probióticos pode ser arriscada nesses pacientes, por conta da elevada imunossupressão à que são submetidos 5 . Diante disso, a presente revisão teve como objetivo identificar as evidências científicas atuais relacionadas à segurança e ao benefício da utilização de probióticos em pacientes submetidos ao TCTH.

\section{MÉTODO}

Este estudo foi elaborado em formato de revisão integrativa de literatura e desenvolvido de acordo com as seguintes etapas: elaboração de uma pergunta norteadora; definiçáo dos descritores para a busca; busca em base de dados; exclusão de artigos repetidos; definição dos critérios de inclusão e exclusão; análise e interpretação dos resultados. Para direcionamento, foi utilizada a seguinte pergunta: "O uso de probióticos é seguro para os pacientes imunossuprimidos submetidos ao TCTH?”.

Os descritores utilizados para a busca foram definidos por meio de consulta aos Descritores em Ciências da Saúde (DeCS), sendo eles: Probióticos/Probiotics, Transplante de Células-Tronco Hematopoéticas/Hematopoietic Stem Cell Transplantation, Hospedeiro Imunocomprometido/ Immunocompromised Host, Microbioma Gastrointestinal/ Gastrointestinal Microbiome e Imunidade/Immunity. Após a definição dos descritores, foi realizada pesquisa nas bases de dados Literatura Latino-americana e do Caribe em Ciências da Saúde (LILACS) e MEDLINE/PubMed, sendo em inglês no PubMed e em português na LILACS, com o descritor probióticos associado aos descritores transplante de células-tronco hematopoéticas, hospedeiro imunocomprometido, microbioma gastrointestinal e imunidade.

Os critérios de inclusão foram: artigos publicados entre 2000 e 2018, pacientes submetidos a TCTH e intervenção com cepas probióticas. Os critérios de exclusão na seleção dos artigos foram: pacientes submetidos a outros tipos de transplante, estudos com animais e artigos de revisão.

Apesar de não se tratar de uma revisão sistemática, o fluxograma de elegibilidade (Figura 1) foi elaborado conforme o método PRISMA (principais itens para relatar revisóes sistemáticas e metanálises) para facilitar a compreensão da forma como a seleçâo de artigos fora organizada. Esse método consiste em um fluxograma desenvolvido em quatro etapas para determinação de elegibilidade dos artigos ${ }^{6}$.

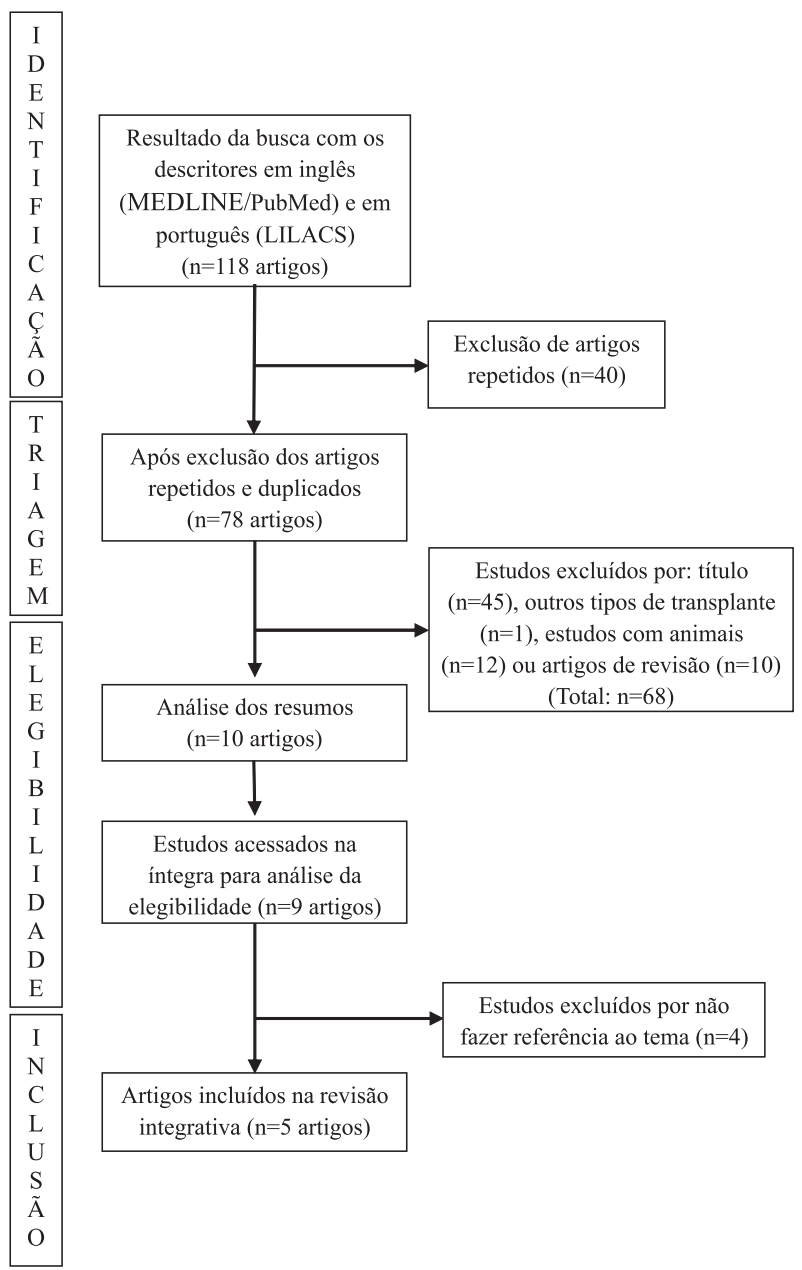

Figura 1. Fluxograma de seleção dos estudos adaptado do método PRISMA 


\section{RESULTADOS}

A seleção dos arquivos disponíveis, conforme os critérios de inclusão e exclusão, resultou em 118 artigos. Destes 118 artigos, resultantes da pesquisa com todas as palavras-chave, 40 artigos se repetiam, sendo então excluídos. Foram então obtidos 78 artigos. Destes, foram excluídos aqueles em que o título não se relacionava com o tema $(n=45)$, outros tipos de transplante $(n=1)$, estudos com animais $(\mathrm{n}=12)$ ou artigos de revisão $(\mathrm{n}=10)$, restando dez artigos. Foram entâo lidos os resumos desses dez artigos e excluídos cinco artigos que não faziam referência ao tema (4) ou não estavam disponíveis na íntegra (1), sobrando cinco artigos para leitura. $\mathrm{O}$ ano de publicação de cada estudo analisado, autores, participantes, modelo do estudo e os resultados estão listados no Quadro $1^{7-9,3,10}$.

Dos artigos elegíveis para análise, foram avaliados, no total, 52 pacientes entre oito meses a 69 anos. Os 52 pacientes eram provenientes dos seguintes países: Estados Unidos e Itália. Quanto ao tipo de transplante, dois pacientes realizaram TCTH autólogo, sendo os demais, halogênico.

Com relação aos tipos de estudo: três eram estudo de caso, um era coorte retrospectivo e um foi um ensaio clínico.

No relato de caso de Cesaro et al. ${ }^{7}$, sobre uma criança de oito meses com diagnóstico de leucemia mieloide aguda (LMA), o paciente estava neutropênico, em tratamento quimioterápico e recebia cápsulas de probiótico com a cepa $S$. boulardii para prevençáo de diarreia associada aos antibióticos. Após o término do segundo ciclo do protocolo ICE, o paciente estava neutropênico e febril. A cepa de $S$. boulardii foi isolada no cateter venoso central (CVC) e foi suspensa a administração do probiótico. Após a retirada do CVC, embora o paciente ainda estivesse neutropênico, não houve mais registro de febre. $\mathrm{O}$ paciente seguiu com quimioterapia e foi submetido ao TCTH. A utilização de cepas de Saccharomyces boulardii pode não ser segura, pois estudos demonstram que algumas cepas probióticas apresentam forte adesão à mucosa intestinal, o que leva ao aumento do risco de translocação bacteriana, entre elas, o $S$. boulardii, sendo que esta não é uma espécie liberada pela Agência Nacional de Vigilância Sanitária (Anvisa) para utilização ${ }^{11}$. Um estudo realizado por Wada et al. ${ }^{12}$ avaliou 42 pacientes submetidos à quimioterapia com doenças malignas, que estavam neutropênicos ou não. Os pacientes foram divididos em dois grupos, um recebeu as cepas probióticas Bifidobacterium breve e outro, placebo. A administração do probiótico e do placebo iniciou duas semanas antes do protocolo de quimioterapia e continuou por mais seis semanas. A utilização de antibiótico parenteral e a frequência e duração da febre foram menores nos pacientes que utilizaram probióticos. Não houve diferença entre os grupos no uso de antibióticos orais, contagem de bactérias e frequência da diarreia. Os autores justificam que não houve diferença na frequência da diarreia, pois a dose utilizada de probiótico $\left(10^{4}-10^{6}\right.$ unidades formadoras de colônia (UFC)/g não foi suficiente para compensar as mudanças na flora bacteriana induzida pela quimioterapia.

No relato de caso de Mehta et al. ${ }^{8}$, foi descrito o caso de um paciente de 69 anos, com diagnóstico de linfoma do manto, estágio III, o qual desenvolveu sepse associada ao consumo excessivo de iogurte enriquecido com probióticos da espécie Lactobacillus acidophilus. O paciente foi submetido ao TCTH autólogo e desenvolveu mucosite severa, vômitos, diarreia e enterorragia. A colonoscopia e a biópsia apresentaram inflamaçôes agudas e crônicas e houve crescimento de Lactobacillus acidophilus na cultura sanguínea. Em relato do paciente, foi evidenciado o consumo de seis a oito copos de iogurte diariamente, enriquecido com cepas de L. Acidophilus. A infecção foi resolvida após a interrupção do consumo do iogurte, sem mudanças nos antibióticos. Os Lactobaccilus podem estar presentes em alimentos como: iogurtes, queijos, chucrute e outros fermentados. Casos de infecçôes e complicaçôes são bastante raros no uso de Lactobacillus, porém os pacientes imunocomprometidos são mais vulneráveis a infecçôes ${ }^{13}$.

Cohen et al. ${ }^{9}$ avaliaram a ocorrência de infecções de corrente sanguínea causadas por probióticos nos pacientes submetidos ao TCTH em um centro em Seattle, Washington. Foi revisado o banco de dados desse centro com dados entre 2002 e 2011. Os pacientes não foram proibidos de consumir alimentos que potencialmente continham probióticos, como iogurtes. Foi considerada infecção de corrente sanguínea quando o paciente tinha pelo menos uma cultura sanguínea positiva para espécies de Lactobacillus, Bifidobacterium, Streptococcus thermophiles e Saccharomyces no período de um ano após o TCTH. Os óbitos foram considerados atribuíveis ao probiótico caso ocorressem dentro de 14 dias do isolamento de uma cepa probiótica em cultura e se os pacientes tivessem sinais ou sintomas antecedentes compatíveis. Foram elegíveis para avaliação 3.796 pacientes. Destes, 19 pacientes $(0,5 \%)$ desenvolveram infecção de corrente sanguínea dentro de um ano após o TCTH. Dos 19 pacientes, 18 tiveram bacteremia relacionada a Lactobacillus, o que ocorreu em média 84 dias após o TCTH. Dos 19 pacientes, 17 deles tiveram apenas uma cultura positiva, porém, um paciente apresentou bacteremia prolongada por Lactobacillus ( 25 culturas positivas consecutivas) e outro, fungemia prolongada por Saccharomyces (5 culturas positivas consecutivas). Oito pacientes (44\%) foram diagnosticados com doença do enxerto contra hospedeiro (DECH) intestinal antes do desenvolvimento de bacteremia. Dos 
Quadro 1. Descrição dos estudos utilizados na revisão

\begin{tabular}{|c|c|c|c|c|c|}
\hline 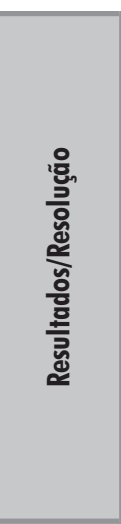 & 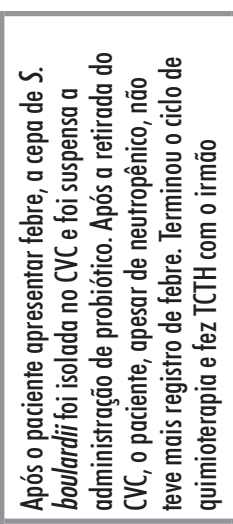 & 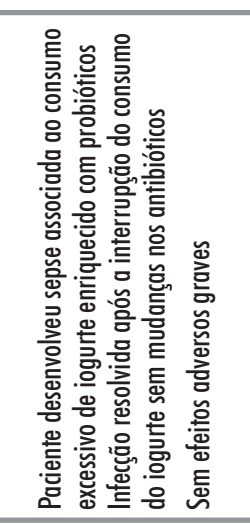 & 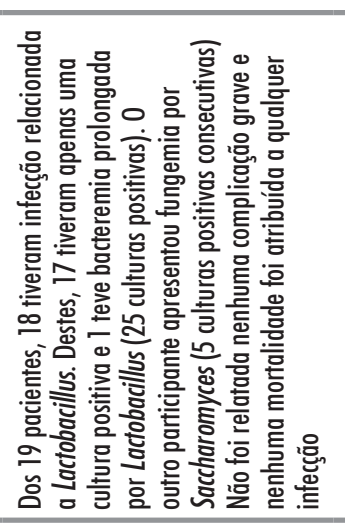 & 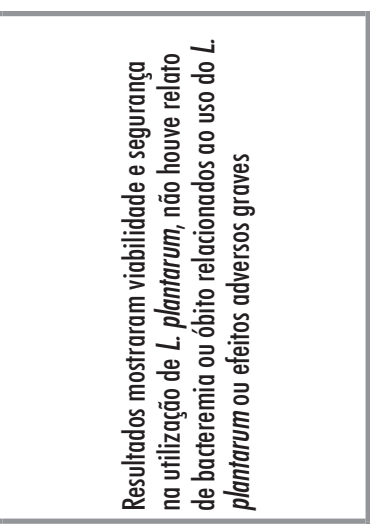 & 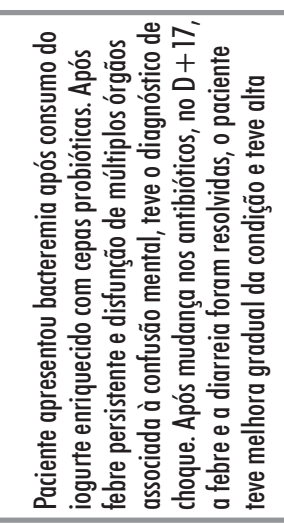 \\
\hline 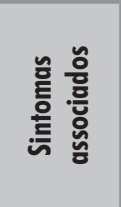 & 嬊 & 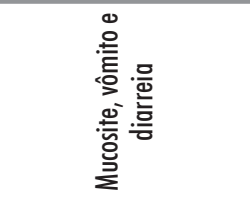 & 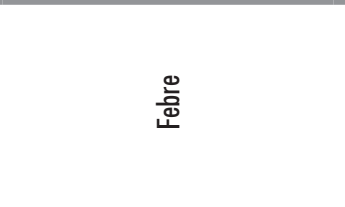 & 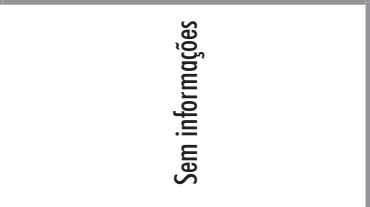 & 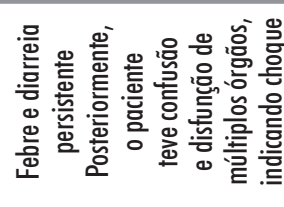 \\
\hline 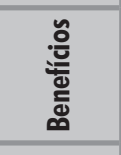 & 惫 & 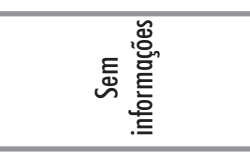 & 惫 & 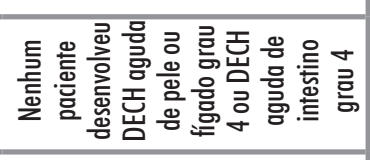 & ) \\
\hline 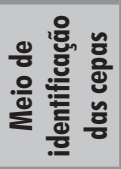 & $\breve{\Xi}$ & 亳总 & 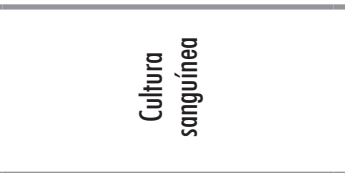 & 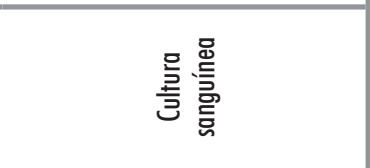 & 礠总言 \\
\hline 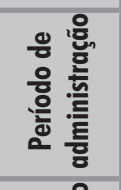 & 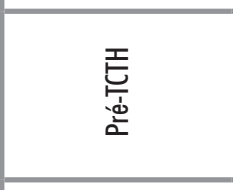 & 里 & 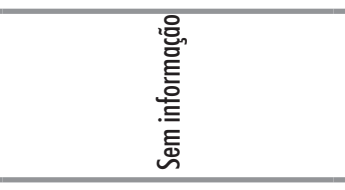 & 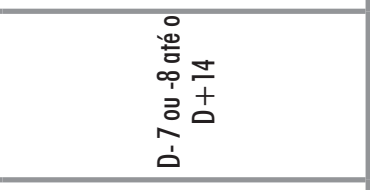 & 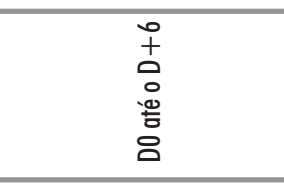 \\
\hline 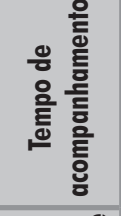 & 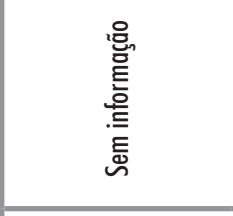 & 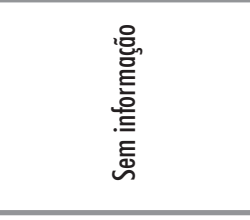 & 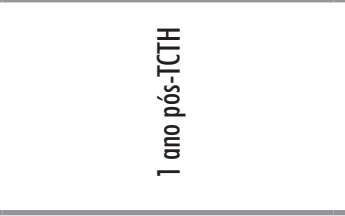 & 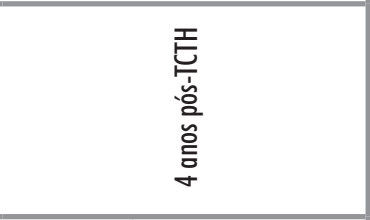 & 递 \\
\hline 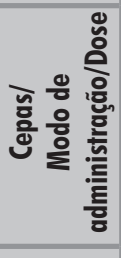 & 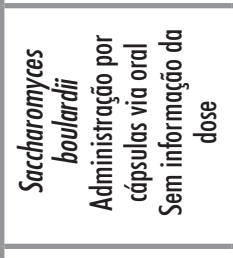 & 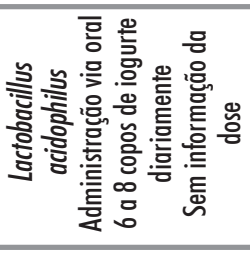 & 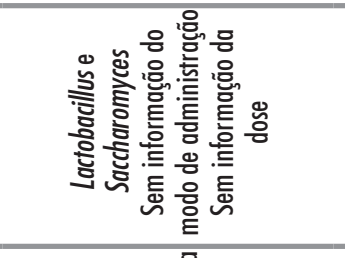 & 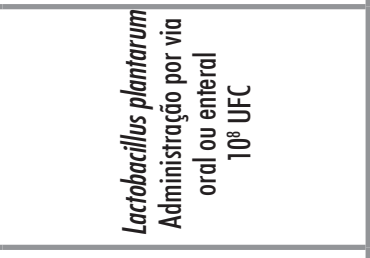 & 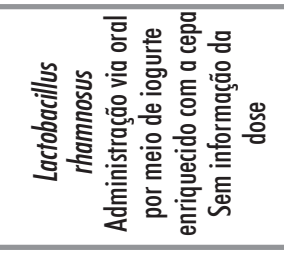 \\
\hline$\stackrel{\circ}{=}$ & 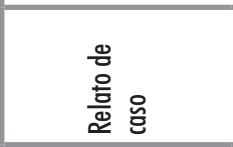 & $\begin{array}{l}\stackrel{0}{0} \\
\text { o } \\
\frac{0}{2}\end{array}$ & 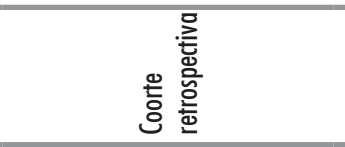 & 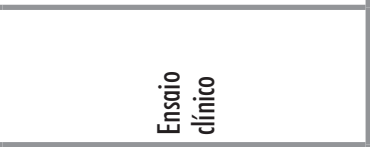 & 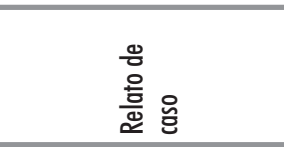 \\
\hline 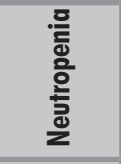 & 结 & हE & 总 & E & E \\
\hline$\frac{\frac{8}{3}}{\frac{\bar{a}}{2}}$ & 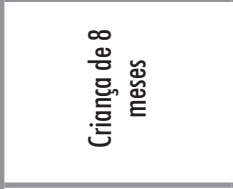 & 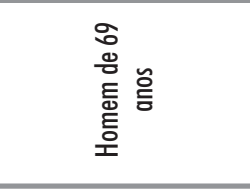 & 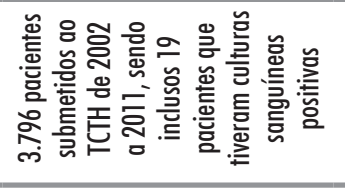 & 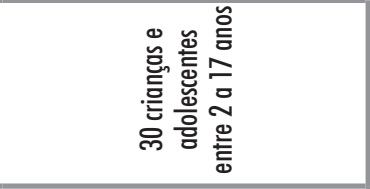 & 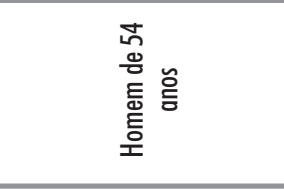 \\
\hline 言是 & 응 言咅 & 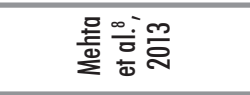 & 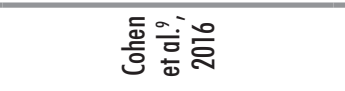 & 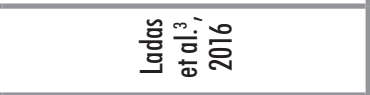 & 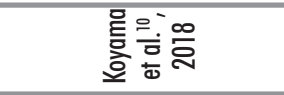 \\
\hline
\end{tabular}

Legendas: CVC: Cateter Venoso Central; DECH: Doença do Enxerto contra Hospedeiro; TCTH: Transplante de Células-Tronco Hematopoiéticas; UFC: Unidades Formadoras de Colônia. 
17 pacientes com apenas uma cultura positiva, quatro apresentaram sintomas, sendo a febre o sintoma mais comum. Não foi relatada nenhuma complicação infecciosa grave e nenhuma mortalidade foi atribuída a essas infecções. Os autores ressaltam que o desenvolvimento de bacteremia nos pacientes estudados foi pouco frequente e que, naqueles que a desenvolveram, nenhuma morte foi diretamente atribuída a isso. A maioria desses eventos ocorreu antes do $\mathrm{D}+100$, período em que ocorre o maior pico de imunossupressáo. Os autores concluem que os probióticos incorporados a produtos alimentícios parecem ser causa rara de infecçôes de corrente sanguínea e estáo associados à baixa mortalidade.

No estudo realizado por Ladas et al. ${ }^{3}$, foram utilizadas cepas de L. plantarum com finalidade probiótica em 30 crianças e adolescentes de 2 a 17 anos, avaliados durante um período de quatro anos. Os pacientes receberam a quantidade de $1 \times 10^{8} \mathrm{UFC}$, por via oral, do D -8 ou -7 até o D+14. Náo houve relatos de bacteremia relacionada ao uso de Lactobacillus ou efeitos adversos graves dessa intervenção. Três pacientes inclusos no estudo foram a óbito antes do $\mathrm{D}+100$, no entanto, nenhuma dessas mortes foi relacionada ao uso do probiótico. Ainda neste estudo, foi verificado que $70 \%$ dos pacientes não desenvolveram DECH intestinal aguda até o D+100, o que pode ser um benefício da administração dos probióticos. Ao final, apesar de os resultados terem sido seguros e viáveis, os autores reforçam a recomendação de não utilizar probióticos como rotina, com base nos resultados de seu estudo.

O estudo de Koyama et al. ${ }^{10}$ descreve o caso de um paciente do sexo masculino, 54 anos, com leucemia promielocítica. O paciente teve remissáo completa da doença seguida de quimioterapia em alta dose e posterior TCTH autólogo. Houve a ocorrência de diarreia grave desde o D0, além da utilização de cefepime, o paciente utilizou voluntariamente iogurte enriquecido com $L$. rhamnosus até o D+6. Cada copo do iogurte continha mais de 14 bilhôes de UFC. No D+8, o paciente estava com febre alta, além de diarreia grave persistente, com identificação negativa de $C$. difficile nas fezes. No D+10, além da febre e diarreia, o paciente apresentou confusão mental e disfunção múltipla de órgãos, indicando choque. Após administração de altas doses de ampicilina, no $\mathrm{D}+17$, a febre e a diarreia foram resolvidas, a condição geral do paciente melhorou e ele teve alta.

\section{DISCUSSÃO}

Os estudos disponíveis que avaliam o uso de probióticos em pacientes submetidos ao TCTH são escassos e, até o momento, não permitem a realização de revisão sistemática. Assim, dada a relevância do tema, realizou-se uma revisão integrativa trazendo uma análise mais abrangente do tema, a fim de oferecer subsídios para futuros ensaios clínicos controlados.

Para ser considerado um probiótico, o micro-organismo deve atender a alguns quesitos: 1) ser de origem humana; ou seja, ser reconhecido pelo sistema imunológico intestinal também conhecido como gut-associated lymphoid tissue/tecido linfático associado ao intestino (GALT) como integrante natural da microbiota humana; 2) não apresentar patogenicidade (deve ser benéfico ao intestino e não estimular doenças); 3) ser resistente ao processo de produção de alimentos; 4) seu uso deve permanecer viável após o contato com suco gástrico e bile; 5) deve apresentar adesão à célula epitelial intestinal; 6) ter capacidade de persistir no trato gastrointestinal; e 7) influenciar a atividade metabólica local ${ }^{5}$. Além dessa caracterização, o uso de probióticos deve ter a aprovação de agências sanitárias reguladoras e cujos critérios estabelecidos podem ser diversos entre países.

Em atualização às normas publicadas anteriormente, a Anvisa ${ }^{11}$ aprova atualmente as seguintes espécies comprovadamente benéficas ao ser humano: Bacillus coagulans GBI-30, Bifidobacterium lactis HN019 e Lactobacillus reuteri DSM 17938. Se pode notar que, dos estudos analisados nesta revisão, nenhuma cepa seria passível de ser utilizada para a populaçáo brasileira, com falta de dados científicos em âmbito nacional que justifiquem a utilização. Considerando que iogurtes e outros produtos com probióticos estão amplamente disponíveis aos consumidores, o trabalho traz uma perspectiva de risco pelo uso náo orientado desses produtos.

A bacteremia associada ao uso de probióticos, especialmente em pacientes imunossuprimidos, é um risco e, no estudo de Koyama et al. ${ }^{10}$, a septicemia causada pela ingestão voluntária de iogurtes disponíveis comercialmente levou ao quadro de choque, assim, não só os benefícios, mas os riscos do uso de probióticos são fatores relevantes a serem considerados quanto à administração de probióticos para pacientes submetidos ao TCTH, fator relevante a ser considerado. De modo geral, os probióticos são considerados seguros, tendo em vista sua ampla utilizaçáo na indústria de alimentos. No entanto, também são relatados alguns efeitos graves como infecçôes ou mesmo sepse ${ }^{13}$.

Outro risco associado ao uso de probióticos esteve presente no relato de caso de Cesaro et al. ${ }^{7}$ no que diz respeito à contaminaçáo cruzada que ocorreu no CVC. Em uma revisão que identificou casos de bacteremia por Saccharomyces boulardii ${ }^{14}$ até 2007, foi observado que os pacientes afetados eram portadores de CVC. Além disso, 
alguns pacientes não utilizaram compostos à base de $S$. boulardii, mas entraram em contato com pacientes que estavam utilizando, portanto, a contaminaçáo pode ter ocorrido por meio das máos dos cuidadores ou pelo ar na abertura dos sachês ou cápsulas.

Ainda são controversos os resultados encontrados na literatura quanto ao uso de probióticos em pacientes imunocomprometidos. Um estudo realizado por Esaiassen et al. ${ }^{15}$ constatou bacteremia em pacientes pediátricos imunocomprometidos que utilizaram cepas de Bifidobacterium. Um estudo realizado por Wolf et al. ${ }^{16}$ com pacientes HIV positivos e imunocomprometidos mostrou que o uso de Lactobacillus reuteri foi seguro e bem tolerado pelos pacientes, não havendo nenhuma intercorrência durante o uso do probiótico, porém não houve diferença significativa na diminuição do número de evacuaçôes diarreicas nesses pacientes. Ressalta-se, no entanto, a neutropenia importante que ocorre em pacientes submetidos ao TCTH e deve ser levada em consideração.

Em uma revisão sistemática realizada por Wei et al. ${ }^{17}$, foram analisados 12 estudos com 1.554 participantes, os autores concluíram que há evidências limitadas para sustentar o uso de probióticos na prevenção ou tratamento da diarreia relacionada à radioterapia (em conjunto ou não com quimioterapia). Os estudos inclusos apresentaram baixo impacto e alta heterogeneidade, ainda que não tenha ocorrido efeito adverso grave. Cabe ressaltar que a mencionada revisão sistemática trata de estudo em pacientes em uso de radioterapia para tratamento de câncer e que a condição clínica de pacientes submetidos ao TCTH, objeto da presente revisão, é singular, uma vez que os pacientes são submetidos a um regime intenso de condicionamento, o que leva a uma imunossupressão mais agressiva em relação ao tratamento do câncer de uma forma geral.

O único estudo clínico realizado com pacientes neutropênicos submetidos ao TCTH, com base nos critérios desta revisão integrativa, foi o estudo de Ladas et al. ${ }^{3}$, no qual probióticos foram utilizados tanto via oral quanto via enteral e o uso se mostrou seguro. Embora nenhum paciente tenha desenvolvido $\mathrm{DECH}$ de pele ou fígado grau 4 ou DECH aguda de intestino grau 4, não ficou claro no estudo se este seria um benefício da utilização das cepas probióticas. Além disso, o estudo apresentou algumas limitações como a ausência de grupo controle e cegamento e número reduzido de participantes. Em abril de 2014, foi aprovada uma alteração que permitia a inclusão de pacientes que estivessem em uso de levofloxacino e de outros probióticos, critérios estes que podem comprometer a qualidade do estudo e seus resultados.
O Lactobacillus plantarum, utilizado no trabalho de Ladas et al. ${ }^{3}$, tem sido utilizado em grande escala em infecçóes intestinais e na diminuição do risco de diarreia associada ao uso de antibióticos ${ }^{7}$. Uma revisão realizada por Hemaiswarya et al. ${ }^{18}$ mostrou que o uso de L. plantarum está relacionado com a modulação da microbiota intestinal e na integralidade do epitélio. Além disso, está envolvido na exclusão de patógenos e na secreção de peptídeos antimicrobianos.

Nos demais trabalhos, incluindo o estudo prospectivo de Cohen et al. ${ }^{9}$, os sintomas relacionados ao uso de probióticos foram registrados como: febre, vômito e diarreia, apesar de não estarem necessariamente associados ao uso desse produto. No trabalho de Cohen et al. ${ }^{9}$, um número maior de indivíduos foi avaliado, no entanto, a ausência de algumas informaçôes importantes para compreensão da intervenção limitam sua análise crítica. Não foi possível identificar, por exemplo, se os pacientes estavam neutropênicos, o período de utilizaçáo dos probióticos ou produtos contendo probióticos, bem como a dose e via de utilização.

Outro aspecto importante a destacar é que não foi possível identificar na literatura uma homogeneidade nas metodologias empregadas quanto ao desenho científico e à especificidade para pacientes submetidos ao TCTH. São encontrados com mais frequência estudos em pacientes em quimioterapia ou imunodeprimidos náo relacionados ao condicionamento para transplante e com diversas espécies de bactérias ou misturas de espécies, em quantidades diversas, frequência e limites de consumo distintos. Além disso, as características clínicas dos pacientes podem ser bastante heterogêneas, dificultando ainda mais a definição de um padrão a ser utilizado em estudos clínicos e a análise dos efeitos da segurança do uso de probióticos.

De maneira geral, a quantidade proposta para administração de probióticos de modo que proporcionem benefícios à saúde é de cinco bilhôes $\left(5 \times 10^{9}\right)$ de UFC por dia, por pelo menos cinco dias, embora alguns estudos que avaliam os efeitos benéficos da utilização de probióticos preconizem doses entre $10^{6}$ a $10^{9} \mathrm{UFC}^{17}$.

Os estudos de Ladas et al. ${ }^{3}$, Mehta et al. ${ }^{8}$ e Cesaro, et al. ${ }^{7}$, que informaram os períodos em que foram administrados os probióticos (D -8 ou -7; D+10 e préTCTH, respectivamente), se enquadram no período proposto para trazer benefícios ao paciente. No Brasil, a quantidade mínima diária de ingestão de probióticos é fixada em $10^{8}$ a $10^{9}$ UFC por $\operatorname{dia}^{14}$ para a populaçáo saudável. No entanto, conforme já mencionado, em razão da escassez de estudos com pacientes imunossuprimidos que passaram pelo TCTH, é incerto afirmar a dose segura para utilização nesse público. 
Dois aspectos podem aqui ser destacados: o efeito clínico benéfico do uso de probióticos e a segurança do seu uso em pacientes imunossuprimidos. Nos artigos avaliados nesta revisão, os benefícios na utilização de probióticos em pacientes submetidos ao TCTH não ficaram claros. Independentemente da formulação a ser utilizada, um ponto fundamental na administração das cepas é o manuseio seguro dos probióticos. Recomenda-se o uso de luvas no manuseio, preparo e administração das cepas probióticas, que devem ser descartadas após a utilização, seguido da lavagem das mãos. Essa prática visa a minimizar a exposição de outros pacientes às cepas probióticas. Além disso, os profissionais de saúde devem estar cientes do risco de contaminação pelo CVC e acessos periféricos enquanto há manipulaçâo do probiótico. A abertura de sachês contendo as cepas deve ser feita longe de pacientes, em um espaço restrito para evitar a formaçáo de aerossol de esporos e a contaminação de locais estéreis. Portanto, recomenda-se que sejam utilizadas cápsulas em detrimento dos sachês, pois há menor risco de contaminaçăo ${ }^{19}$.

Mesmo para a construção de uma revisão integrativa, os trabalhos disponíveis não foram suficientes para nortear o entendimento sobre o uso de probióticos para paciente submetidos ao TCTH; entretanto, cabe aqui destacar que se trata de um campo para futuras pesquisas para a avaliação dos efeitos e da segurança do uso de probióticos nesse caso. Apesar de não ter sido possível indicar um posicionamento quanto aos achados dos estudos avaliados nesta revisão, destaca-se como limitação importante a falta de análise do risco de viés dos estudos encontrados.

\section{CONCLUSÃO}

Embora seja um tema de atual interesse na área, ainda são escassas as evidências que possam identificar riscos e benefícios; doses e modo de administração de probióticos em pacientes submetidos ao TCTH.

Destaca-se que, nos estudos avaliados, apesar de não terem sido encontrados efeitos adversos graves ou óbitos decorrentes da utilização de probióticos, houve registro de bacteremia em pacientes que utilizaram cepas com finalidade probiótica. Os benefícios que, porventura, tenham sido observados em outras populaçóes de indivíduos imunodeprimidos náo foram observados nos estudos incluídos nesta revisão, portanto, são necessários mais estudos a fim de constatar os efeitos da utilização dos probióticos em pacientes submetidos ao TCTH.

\section{CONTRIBUIÇÕES}

Paola Dantas Pinheiro de Oliveira contribuiu na concepção, na redação e na aprovação final da versão para publicação. Denise Johnsson Campos e Vaneuza Araújo Moreira Funke contribuíram na concepção, na revisão crítica com contribuição intelectual e na aprovação final da versão para publicação. Sarah Mehl Coradi contribuiu na redação e na aprovação final da versão para publicação. Regina Maria Vilela contribuiu no desenho do trabalho, na revisão crítica com contribuição intelectual e na aprovação final da versão para publicação.

\section{DECLARAÇÃO DE CONFLITOS DE INTERESSE}

Nada a declarar.

\section{FONTES DE FINANCIAMENTO}

Não há.

\section{REFERÊNCIAS}

1. Staffas A, Burgos da Silva M, van den Brink MR. The intestinal microbiota in allogeneic hematopoietic cell transplant and graft-versus-host disease. Blood. 2017;129(8):927-33. doi: https://doi.org/10.1182/ blood-2016-09-691394

2. Paix A, Antoni D, Waissi W, et al. Total body irradiation in allogeneic bone marrow transplantation conditioning regimens: a review. Crit Rev Oncol Hematol. 2018;123:138-48. doi: https://doi.org/10.1016/j. critrevonc.2018.01.011

3. Ladas EJ, Bhatia M, Chen L, et al. The safety and feasibility of probiotics in children and adolescents undergoing hematopoietic cell transplantation. Bone Marrow Transplant. 2016;51(2):262-6. doi: https://doi. org/10.1038/bmt.2015.275

4. Zama D, Biagi E, Masetti R, et al. Gut microbiota and hematopoietic stem cell transplantation: where do we stand? Bone Marrow Transplant. 2017;52(1):7-14. doi: https://doi.org/10.1038/bmt.2016.173

5. Zawistowska-Rojek, A, Tyski, S. Are probiotic really safe for humans? Pol J Microbiol. 2018;67(3):251-8. doi: https://doi.org/10.21307/pjm-2018-044

6. Moher D, Liberati A, Tetzlaff J, et al. Preferred reporting items for systematic reviews and meta-analyses: the PRISMA statement. PLoS Med. 2009;6(7):e1000097. doi: https://doi.org/10.1371/journal.pmed.1000097

7. Cesaro S, Chinello P, Rossi L, et al. Saccharomyces cerevisiae fungemia in a neutropenic patient treated with Saccharomyces boulardii. Support Care Cancer. 2000;8(6):504-5. doi: http://dx.doi.org/10.1007/ s005200000123

8. Mehta A, Rangarajan S, Borate U. A cautionary tale for probiotic use in hematopoietic SCT patientsLactobacillus acidophilus sepsis in a patient with mantle cell lymphoma undergoing hematopoietic SCT. Bone 
Marrow Transplant. 2013;48(3):461-2. doi: http:// dx.doi.org/10.1038/bmt.2012.153

9. Cohen SA, Woodfield MC, Boyle N, et al. Incidence and outcomes of bloodstream infections among hematopoietic cell transplant recipients from species commonly reported to be in over-the-counter probiotic formulations. Transpl Infect Dis. 2016;18(5):699-705. doi: http://dx.doi.org/10.1111/tid.12587

10. Koyama S, Fujita H, Shimosato T, et al. Septicemia from Lactobacillus rhamnosus GG, from a probiotic enriched yogurt, in a patient with autologous stem cell transplantation. Probiotics Antimicrob Proteins. 2018;11(1):295-8. doi: http://dx.doi.org/10.1007/ s12602-018-9399-6

11. Agência Nacional de Vigilância Sanitária. Probióticos: construção da lista de linhagens probióticas [Internet]. Brasília (DF): ANVISA; abril. 2007. [acesso 2017 maio 20]. Disponível em: http://portal.anvisa.gov.br/ documents/3845226/0/An\%C3\%A1lise+das+Linhage ns+de+Probi\%C3\%B3ticos_23042018.pdf/6e37da132151-4330-85b0-0f449dbb0e95

12. Wada M, Nagata S, Saito M, et al. Effects of the enteral administration of Bifidobacterium breve on patients undergoing chemotherapy for pediatric malignancies. Support Care Cancer. 2010;18(6):751-9 2010. doi: http://dx.doi.org/10.1007/s00520-009-0711-6

13. Stadlbauer V. Immunosuppression and probiotics: are they effective and safe? Benef Microbes. 2015;6(6):823-8. doi: http://dx.doi.org/10.3920/BM2015.0065

14. Segarra-Newnham M. Probiotics for Clostridium difficile-associated diarrhea: focus on Lactobacillus rhamnosus GG and Saccharomyces boulardii. Ann Pharmacother. 2007;41(7):1212-21. doi: https://doi. org/10.1345/aph.1K110

15. Esaiassen E, Hjerde E, Cavanagh JP, et al. Bifidobacterium bacteremia: clinical characteristics and a genomic approach to assess pathogenicity. J Clin Microbiol. 2017;55(7):2234-48. doi: http://dx.doi.org/10.1128/ JCM.00150-17

16. Wolf BW, Wheeler KB, Ataya DG, et al. Safety and tolerance of Lactobacillus reuteri supplementation to a population infected with the human immunodeficiency virus. Food Chem Toxicol. 1998;36(12):1085-94. doi: http://dx.doi.org/10.1016/s0278-6915(98)00090-8

17. Wei D, Heus P, van de Wetering FT, et al. Probiotics for the prevention or treatment of chemotherapy- or radiotherapy-related diarrhoea in people with cancer. Cochrane Database Syst Rev. 2018;8:CD008831. doi: http://dx.doi. org/10.1002/14651858.CD008831.pub3

18. Hemaiswarya S, Raja R, Ravikumar R, et al. Mechanism of action of probiotics. Braz Arch Biol Technol. 2013;56(1):113-9. doi: http://dx.doi.org/10.1590/ S1516-89132013000100015
19. Urben LM, Wiedmar J, Boettcher E, et al. Bugs or drugs: are probiotics safe for use in the critically Ill? Curr Gastroenterol Rep. 2014;16(7):388. doi: http://dx.doi. org/10.1007/s11894-014-0388-y

Recebido em 27/6/2019

Aprovado em 21/11/2019 